\title{
Aortoenteric Fistula: Endovascular Treatment
}

\author{
Umberto G Rossi MD ${ }^{1}$ and Francesco Petrocelli MD² \\ 'Department of Diagnostic Imaging - Interventional Radiology Unit, E.O. Galliera Hospital, Mura delle Cappuccine 14, \\ 16128 Genova, Italy \\ 2 Department of Radiology and Interventional Radiology, IRCCS San Martino Policlinic University Hospital, \\ Largo Rosanna Benzi 10, 16132 Genova, Italy
}

Keywords: Aorta; Fistula; Endovascular; Balloon; Endoprosthesis

Received: 28 January 2021; Accepted: 8 February 2021

A 67-year-old male patient arrived at our emergency department with abdominal pain and near syncope status: blood pressure $90 / 50 \mathrm{mmHg}$ and heart rate 80 beats $/ \mathrm{min}$. His past medical history revealed an aorto-biliac surgical graft for abdominal aortic aneurysm (3 years ago), hypertension and left lower-leg claudication. Abdominal examination revealed no palpable mass. He underwent urgent, abdominal multi-detector computed tomography (MD-CT), with axial (Figure $1 a)$ and coronal (Figure 1b) VRT reconstruction, which demonstrated, in the arterial unenhanced phase, a direct contrast extravasation (arrowhead) from the middle-distal third of the abdominal aorta to a small bowel loop (*) due to a fissured anastomotic pseudoaneurysm. Occlusion of the left common iliac artery (\#) was also noted (Figure $1 b$ ).

The patient, who had already received and been maintained for 1 month on empiric antibiotic therapy (vancomycin, piperacillin, and tazobactam), was transferred immediately to an angiography room. From the left brachial and right femoral arteries, a 10-Fr and 20-Fr introducer, respectively, was inserted. From the left brachial access, an occlusion balloon was positioned and inflated in the abdominal aorta in front of the renal artery origins

\section{Corresponding author:}

Umberto G. Rossi, Department of Diagnostic Imaging Interventional Radiology Unit, E.O. Galliera Hospital, Mura delle Cappuccine 14, 16128 Genova, Italy.

Email: urossi76@hotmail.com, umberto.rossi@galliera.it

(C) 2021 CC BY 4.0 - in cooperation with Depts. of Cardiothoracic/ Vascular Surgery, General Surgery and Anesthesia, Örebro University Hospital and Örebro University, Sweden (arrowhead; Figure $2 a$ ). With the occlusion balloon still inflated (as a shadow image - arrowhead), an endovascular aorta-aortic endoprosthesis (Medtronic, Minneapolis, MN, USA) was deployed from the right femoral access in the middle-distal third of the abdominal aorta (markers - arrows; Figure 2b), with the aim of covering the fissured anastomotic pseudoaneurysm. Final abdominal aorta angiographic control (Figure 2c) demonstrated the correct positioning of the endoprosthesis and exclusion of the fissured aortic pseudoaneurysm. In this case, endovascular aorta-aortic endoprosthesis was a definitive treatment with observed clinical and technical successes on a 2-year follow-up.

\section{Ethics Statement}

(1) All the authors mentioned in the manuscript have agreed to authorship, read and approved the manuscript, and given consent for submission and subsequent publication of the manuscript.

(2) The authors declare that they have read and abided by the JEVTM statement of ethical standards including rules of informed consent and ethical committee approval as stated in the article.

\section{Conflicts of Interest}

The authors declare that they have no conflicts of interest.

\section{Funding}

The authors received no financial support for the research, authorship, and/or publication of this article. 

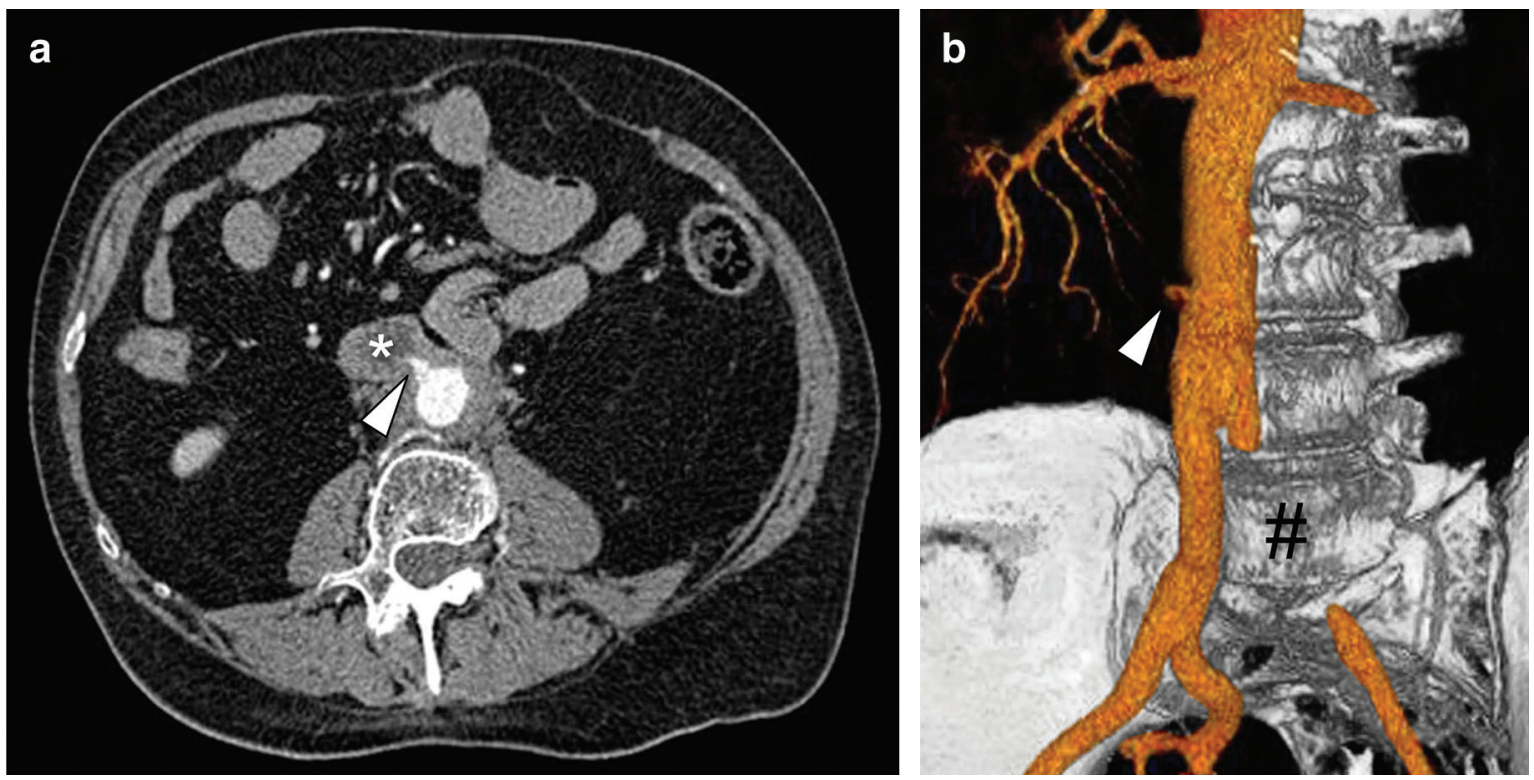

Figure 1
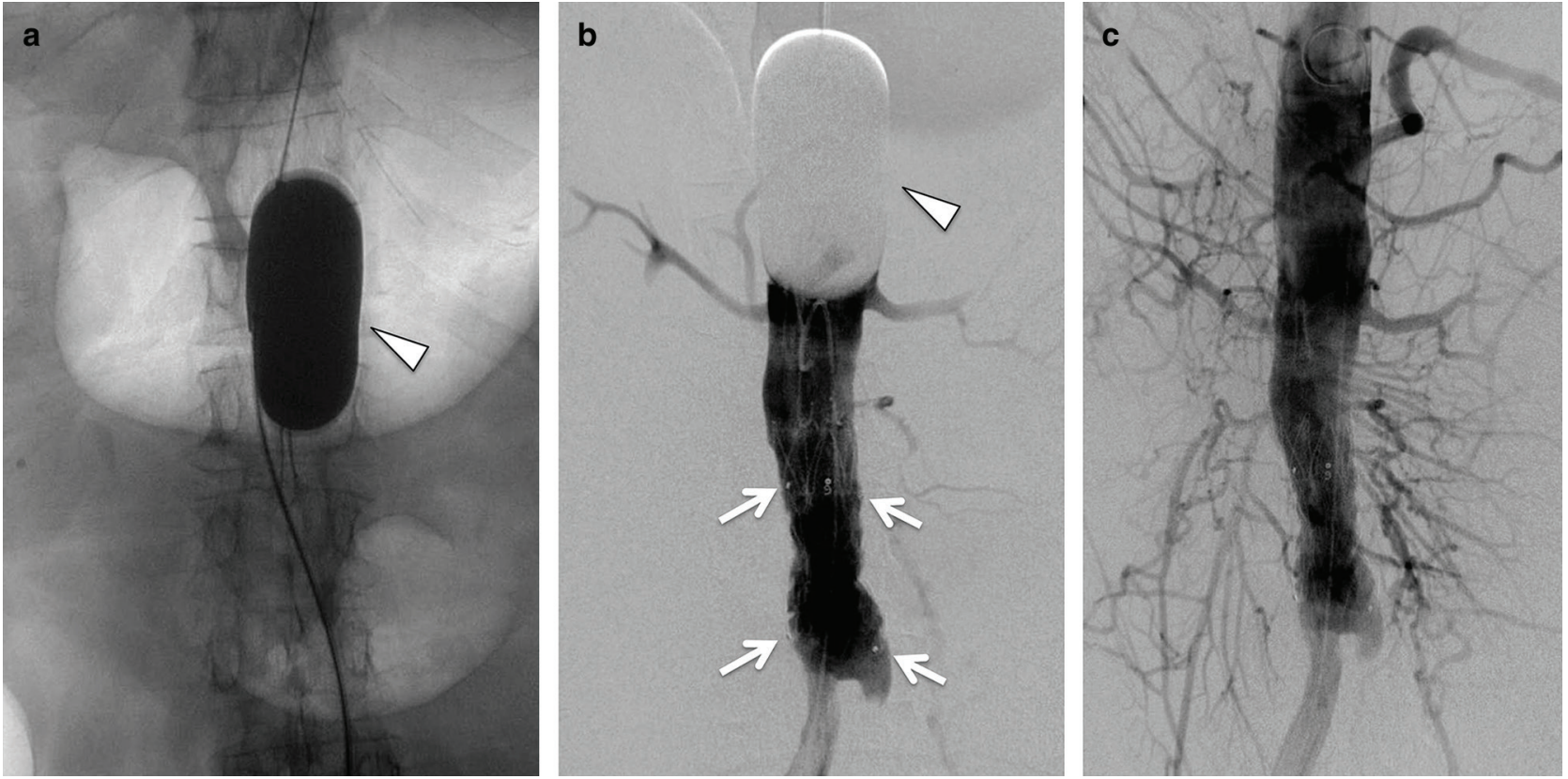

Figure 2 